\title{
Optimal Power Market Participation of Plug-In Electric Vehicles Pooled by Distribution Feeder
}

\begin{tabular}{|r|l|}
\hline Journal: & IEEE Transactions on Power Systems \\
\hline Manuscript ID: & TPWRS-00431-2011.R3 \\
\hline Manuscript Type: & Transactions \\
\hline Date Submitted by the Author: & $\mathrm{n} / \mathrm{a}$ \\
\hline Complete List of Authors: & $\begin{array}{l}\text { Foster, Justin; Boston University, Systems Engineering } \\
\text { Caramanis, Michael; Boston University, Systems Engineering }\end{array}$ \\
\hline Technical Topic Area : & Power system markets, Power system economics \\
\hline Key Words: & $\begin{array}{l}\text { Load management, Electric vehicle grid integration, Wholesale power } \\
\text { markets, Distribution network, Dynamic programming, Scenario tree }\end{array}$ \\
\hline
\end{tabular}

\section{SCHOLARONE \\ Manuscripts}




\title{
Optimal Power Market Participation of Plug-In Electric Vehicles Pooled by Distribution Feeder
}

\author{
Justin M. Foster, Graduate Student Member, IEEE, and Michael C. Caramanis, Member, IEEE
}

\begin{abstract}
Electric vehicle grid integration has the potential to stress distribution network equipment and increase peak consumption, unless properly managed. In this paper, we use dynamic programming to develop a decision support algorithm and market participation policy for a load aggregator (LA) managing the charging of plug-in electric vehicles (PEVs) connecting at the same distribution network feeder. The LA submits inflexible and flexible bids to a liberalized hour-ahead power market, while monitoring localized feeder and PEV rate constraints. Flexible bids, which include a bid price or utility, can be cleared as regulation service, cleared as energy, or rejected by the market operator. These market events are probabilistically included within the modeling framework. A case study, based on New York independent system operator data, found that the market participation policy may reduce daily electricity costs for PEVs significantly more than is expected through forecasted electricity price based scheduling.
\end{abstract}

Index Terms-Load management, electric vehicle grid integration, wholesale power markets, distribution network, dynamic programming, scenario tree, decision-making.

\section{NOMENCLATURE}

In order to best present the complex notation necessary to properly formulate this problem, we have adopted the following general convention: indices appear as subscripts and abbreviations of verbal descriptions appear as superscripts.

\section{A. Indices}

$t \quad$ Hour-ahead market period $t=1,2, \ldots, 24(1 \mathrm{~h})$.

$b \quad$ Market bid $b$.

$g \quad$ Generator $g$.

$\tau \quad$ Departure class $\tau=1,2, \ldots, 48$.

$k \quad$ Iteration $k$ of extension period estimation algorithm.

\section{B. Parameters}

$c_{t g} \quad$ Marginal cost of generator $g$ in period $t(\$$ per $\mathrm{kWh})$.

$\underline{p}_{t g} \quad$ Technical min for generator $g$ in period $t(\mathrm{~kW})$.

$\bar{p}_{t g} \quad$ Technical max for generator $g$ in period $t(\mathrm{~kW})$.

$\underline{s} \quad$ Minimum system regulation service (RS) requirement $(\mathrm{kW}$ per $\mathrm{h})$.

$\Delta x_{t \tau}$ Uncharged energy of $\tau$ class plug-in electric vehicles (PEVs) connecting during period $t(\mathrm{kWh})$.

$\Delta n_{t \tau}$ Number of $\tau$ class PEVs connecting during period $t$.

$C_{t} \quad$ Available feeder capacity in period $t(\mathrm{~kW})$.

Manuscript received May 11, 2011; revised November 11, 2011; revised June 1, 2012, revised September 13, 2012. This work was supported by NSF EFRI 1038230, an EPA STAR Fellowship, and a Switzer Fellowship.

Both authors are with the Division of Systems Engineering, Boston University College of Engineering, Boston, MA, 02446 USA (e-mail: jfoster2@bu.edu; mcaraman@bu.edu). $\bar{r} \quad$ Maximum vehicle charging rate $(\mathrm{kW})$.

$\phi \quad$ Penalty cost for uncharged energy (\$ per $\mathrm{kWh})$.

$\mu_{\tau} \quad$ Class $\tau$ extension period uncharged energy cost (\$ per $\mathrm{kWh})$.

\section{State and Decision Variables}

$p_{t g} \quad$ Dispatch of generator $g$ in period $t(\mathrm{kWh})$.

$s_{t g} \quad$ RS cleared for generator $g$ in period $t(\mathrm{~kW}$ per h).

$q_{t b} \quad$ Dispatch of flexible bid $b$ in period $t(\mathrm{kWh})$.

$s_{t b} \quad$ RS cleared for flexible bid $b$ in period $t(\mathrm{~kW}$ per h).

$Q_{t b}^{F} \quad$ Quantity of flexible bid $b$ for period $t(\mathrm{kWh})$.

$\pi_{t b} \quad$ Bid price of flexible bid $b$ in period $t$ (\$ per $\mathrm{kWh}$ ).

$Q_{t b}^{I} \quad$ Quantity of inflexible bid $b$ for period $t(\mathrm{kWh})$.

$f_{t \tau} \quad$ Flexible bid quantity allocated to class $\tau$ in $t(\mathrm{kWh})$.

$i_{t \tau} \quad$ Inflexible bid quantity allocated to class $\tau$ in $t(\mathrm{kWh})$.

$x_{t \tau} \quad$ Uncharged energy of class $\tau$ PEVs at period $t(\mathrm{kWh})$.

$n_{t \tau} \quad$ Number of class $\tau$ PEVs at period $t$.

$\mathbf{x}_{t} \quad$ Vector of state variables for period $t$.

$\mathbf{u}_{t} \quad$ Vector of decision variables for period $t$.

\section{Random Variables}

Note that realizations of random variables are denoted without the tilde.

$\tilde{\lambda}_{t}^{E} \quad$ Energy price for period $t(\$$ per $\mathrm{kWh})$.

$\tilde{\lambda}_{t}^{R} \quad$ RS price for period $t(\$$ per $\mathrm{kWh})$.

$\tilde{\lambda}_{t} \quad$ Price vector for period $t$.

$\tilde{m}\left(\pi_{t b}\right)$ Market outcome of flexible bid $b$ for period $t$.

\section{INTRODUCTION}

I NNOVATION in communication, sensing, automation, and computation intelligence is being adopted at an increasing rate by the electric power system while national directives stress the importance of embedding the intelligence necessary to support increased levels of renewable generation and demand response programs, and to promote grid reliability, stability, and economic and environmental sustainability. Increasing amounts of flexible loads, whether existing (e.g., heat pumps, data processing centers) or emerging (e.g., electric vehicles), provide opportunities for demand to actively participate in power markets as encouraged by the US Federal Energy Regulatory Commission [1], given security issues are properly considered [2]. In the US, demand response and the load-side provision of reserves has entered the early stages of adoption [3], [4]. This paper examines the potential inclusion of plug-in electric vehicles (PEVs) in wholesale power markets through a load aggregator (LA).

Electric vehicle grid integration is an area of active research [5], and extensive work has been published. Studies have 
considered the benefits of using PEV batteries as grid resources serving transmission system stability [6], [7] in a high renewable generation future impacting transmission resources and requirements for capacity reserves and load following [8][10]. Many works consider centralized command and control scheduling approaches in order to minimize electricity costs or power plant cycling, coordinate with times of high renewable generation, and provide valley filling [11]-[13]. In addition, impacts on the low voltage distribution network [14]-[17] have been considered.

Recently, mathematical formulations for smart-charging have been proposed. Ma et al. developed a decentralized control algorithm, which responds to estimates of electricity prices to achieve socially optimal overnight valley filling [18]. Work by Rotering and Ilic proposes dynamic programming algorithms based on a forecast of future electricity prices to minimize costs to the PEV owner [19]. In addition, papers have developed algorithms for optimal bidding of LAs managing the charging of PEVs in power markets [20]-[22].

This paper builds on our previous work [20], as we consider the optimal distributed bidding of a LA participating in a liberalized wholesale energy market. The LA manages PEVs connected to a specific feeder and shares cost reduction benefits with the PEV owners. The LA receives smart grid information on feeder specific capacity constraints, and PEV battery state-of-charge (SoC) and desired departure time. The LA bids to the wholesale market to minimize PEV battery charging costs aiming at a user specified SoC for each PEV by its desired departure time, given that it is physically feasible and economically desirable. In this paper, extensions include the optimal designation of bid prices as well as quantities, proof of the convergence of the extension period approximation algorithm, and extensive numerical results and sensitivities obtained as part of a case study using New York independent system operator (NYISO) data.

This work contributes to the existing literature by considering several yet unexplored market features. Rather than having a perfect forecast of energy and reserve prices, we assume that the LA cannot anticipate prices beyond an associated joint probability distribution. Therefore, rather than solely determining optimal bid quantities, the LA also determines associated bid prices representative of the instantaneous PEV charging utility. In addition, we consider regulation service (RS) transactions in a market where RS is a band of up-and-down capacity, as in NYISO [23] and Pennsylvania-Jersey-Maryland regional transmission organization (PJM) [24], rather than two separate commodities RS-up and RS-down, as in California independent system operator (CAISO) and the electric reliability council of Texas (ERCOT). Finally, we propose a scalable modeling framework and a convergent extension period approximation algorithm. The modeling framework limits the state space explosion that results from a fleet PEVs at varied locations and with different departure times, while the extension period estimation allows for proper accounting of PEVs scheduled to depart outside of the modeled horizon.

This paper is organized as follows. Section II presents the hour-ahead power market structure in which the LA participates. Section III formulates the LA optimal market par- ticipation problem as a stochastic dynamic program. Section IV summarizes our solution algorithm based on multistage stochastic programming, and proves algorithm convergence. Sections V and VI provide case study results and sensitivity analyses, respectively. Section VIII concludes.

\section{Power Market Structure}

In this section, we describe the hour-ahead wholesale power market structure. For simplicity we limit our consideration of reserves to $\mathrm{RS}$, which, as previously mentioned, we assume to consist of an up-and-down band of capacity. We assume that the RS signal is energy neutral over the hour, as is true in NYISO [23]. In addition, we discuss demand-side participation in the hour-ahead market, formulate the market clearing optimization, and describe the key market events from the LA perspective.

\section{A. Demand-Side Participation and Market Clearing}

We assume that LAs participate on a par basis with generators in the joint energy and RS market. For each hour-ahead market period $t$ ( $1 \mathrm{~h}$ in length), demand-side market participants submit flexible bids, indexed by $b$, with an associated bid price or utility, namely

$$
Q_{t b}^{F} \text { and } \pi_{t b} \text {, respectively. }
$$

In addition, demand-side participants may submit unpriced inflexible energy bids $Q_{t b}^{I}$, with an implied infinite opportunity cost, which are cleared as energy by the market operator and charged at the energy clearing price.

Generators, indexed by $g$, submit marginal costs as well as technical minima and maxima,

$$
c_{t g}, \underline{p}_{t g}, \text { and } \bar{p}_{t g} \text {, respectively. }
$$

The market operator receives bids from market participants and co-optimizes energy and RS by solving the energy balance and reserve requirements constrained economic dispatch problem. We present a simplified version of the economic dispatch in (1)-(7), where transmission network and ramp rate constraints are omitted. The simplified economic dispatch minimizes the cost of generation and maximizes flexible demand utility (1), subject to energy balance (2), RS requirements (3), generator maximum (4) and minimum (5) limits, and flexible load maximum (6) and minimum (7) limits.

$$
\min _{p_{t g}, q_{t b}, s_{t g}, s_{t b}} \sum_{g} c_{t g} p_{t g}-\sum_{b} \pi_{t b} q_{t b}
$$

subject to

$$
\begin{aligned}
\sum_{g} p_{t g}=\sum_{b} q_{t b}+\sum_{b} Q_{t b}^{I} & \leftrightarrow \quad \lambda_{t}^{E} \\
\sum_{g} s_{t g}+\sum_{b} s_{t b} \geq \underline{s} & \leftrightarrow \quad \lambda_{t}^{R} \\
p_{t g}+s_{t g} \leq \bar{p}_{t g}, \quad & \forall g \\
p_{t g}-s_{t g} \geq \underline{p}_{t g}, \quad \forall g & \\
q_{t b}+s_{t b} \leq Q_{t b}^{F}, \quad \forall b & \\
q_{t b}-s_{t b} \geq 0, \quad & \forall b
\end{aligned}
$$


The solution of the above constitutes the clearing of the hour-ahead market, and determines market clearing prices for energy, $\lambda_{t}^{E}$, and RS, $\lambda_{t}^{R}$, as well as generation levels, $p_{t g}$, consumption quantities, $q_{t b}$, and RS provision by generators, $s_{t g}$, and demand, $s_{t b}$.

By (6) and (7) it is clear that in order to provide RS, the LA must be consuming at a rate strictly between 0 and $Q_{t b}^{F}$.

\section{B. Market Events}

Note that as market competition increases through demandside resources participation, the probability that the flexible bid is on margin becomes very small. Therefore, assuming the flexible bid is not on margin, market clearing results in three possible market events for the LA. The flexible bid can be cleared as RS, cleared as energy, or rejected.

The flexible bid cleared as RS (FCRS) market event is defined in (8)-(9). The average consumption rate is $\frac{1}{2} Q_{t b}^{F}$ and the independent system operator (ISO) can send commands in real-time to modulate consumption in the range $\left[0, Q_{t b}^{F}\right]$. Note that we do not model the tracking of the real-time RS signal in this paper. The price condition in (8), ensures that the LA is compensated for any lost utility which would result from consuming at 0 when $\pi_{t b}<\lambda_{t}^{E}$ or $Q_{t b}^{F}$ when $\pi_{t b}>\lambda_{t}^{E}$. In this market event, the ISO debits the LA's account for energy, $\frac{1}{2} \lambda_{t}^{E} Q_{t b}^{F}$, and credits the account for RS, $\frac{1}{2} \lambda_{t}^{R} Q_{t b}^{F}$.

$$
\begin{aligned}
\left|\lambda_{t}^{E}-\pi_{t b}\right| & \leq \lambda_{t}^{R} \\
q_{t b} & =s_{t b}=\frac{1}{2} Q_{t b}^{F}
\end{aligned}
$$

The flexible bid cleared as energy ( $F C E$ ) market event is defined in (10)-(12). For this market event, the ISO debits the LA's account for energy, $\lambda_{t}^{E} Q_{t b}^{F}$.

$$
\begin{aligned}
\pi_{t b} & >\lambda_{t}^{E}+\lambda_{t}^{R} \\
q_{t b} & =Q_{t b}^{F} \\
s_{t b} & =0
\end{aligned}
$$

The flexible bid rejected $(F R)$ market event is defined in (13)-(14). For this market event, the LA's account is neither credited nor debited.

$$
\begin{aligned}
\pi_{t b} & <\lambda_{t}^{E}-\lambda_{t}^{R} \\
q_{t b} & =s_{t b}=0
\end{aligned}
$$

Before the market clears, the LA does not know the clearing prices; however, it has information available to estimate the joint probability distribution of the clearing prices. The probability and expected value of each market event at the time bids are made can be calculated by integrating the joint density function of $\tilde{\lambda}_{t}^{E}$ and $\tilde{\lambda}_{t}^{R}$ over the area of the market events shown in Fig. 1. Note that these probabilities and expected values are function of the bid price, $\pi_{t b}$. In this paper, we estimate the impact of market clearing events on the LA by performing this integration. Solving an economic dispatch as in (1)-(7) requires that the LA knows every other market participant's bids and hence can anticipate actual clearing prices.

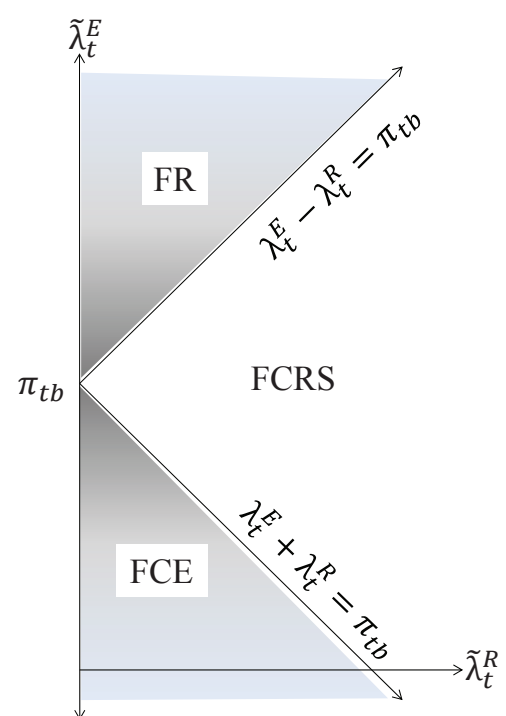

Fig. 1. Graphical depiction of market clearing event areas.

\section{Optimal PEV Charging Decision Support}

We propose a decision support algorithm for optimal bidding to the hour-ahead power market (1)-(7) by a LA who represents PEVs connecting to a specific distribution feeder. In our proposed PEV charging strategy, which we will refer to as the market participation policy, for each market period $t$, the LA submits a single flexible energy bid, $Q_{t b}^{F}$ and $\pi_{t b}$, and a single inflexible energy bid, $Q_{t b}^{I}$, associated with charging vehicles connected to the specific feeder. We model a 24 hour cycle and approximate the infinite horizon by estimating the extension period costs, namely the marginal cost of uncharged PEVs that depart during the next 24 hour cycle.

Since charging decisions are a function of desired departure time, we group PEVs into departure classes $\tau$, which are indicated as subscripts on the decision and state variables. We allocate our bid quantities to individual departure classes, to determine the inflexible (15) and flexible (16) bid quantities introduced above.

$$
\begin{aligned}
Q_{t b}^{I} & =\sum_{\tau} i_{t \tau} \\
Q_{t b}^{F} & =\sum_{\tau} f_{t \tau}
\end{aligned}
$$

\section{A. System Dynamics}

The state of the system is defined by the departure class specific number connected PEVs $\left(n_{t \tau}\right)$ and their uncharged energy $\left(x_{t \tau}\right)$ (in $\mathrm{kWh}$ ). The uncharged energy is calculated using the PEV owner's desired $\mathrm{SoC}$ as

$$
x_{t \tau}=(1-\mathrm{SoC}) * \text { battery capacity. }
$$

The state variables evolve dynamically as a function of energy consumption and new PEV arrivals as shown in (17). The uncharged energy is increased by PEVs plugging-in to the feeder, $\Delta x_{t \tau}$, and decreased by the amount of energy scheduled by the ISO and allocated by the LA. The inflexible bid is automatically cleared; however, the flexible bid is subject to the market events defined in Section II-B. The random 
variable, $\tilde{m}\left(\pi_{t b}\right)$, indicates the impact of market events on the energy capacity dynamics; and therefore, takes a value of $\frac{1}{2}$ if the flexible bid is cleared as RS (FCRS), 1 if the flexible bid is cleared as energy (FCE), and 0 if the flexible bid is rejected (FR). Note that the value of $\frac{1}{2}$ for market event FCRS is based on the expected (or average) consumption, given the energy neutrality assumption with regards to the RS signal.

We assume the cleared energy is assigned by the LA to distribute the uncharged capacity evenly within a departure class. This spreads equally the risk of not fully charging an individual PEV by its scheduled departure time. Thus, when modeling the dynamics for the number of PEVs, we need only consider PEVs plugging-in to the feeder, $\Delta n_{t \tau}$, as shown in (18). In addition, as the maximum charging rate increases this balancing assumption becomes robust. Finally, if the PEV departure class has passed, then the number and uncharged energy is set to zero (19).

$$
\begin{aligned}
x_{(t+1) \tau} & =x_{t \tau}+\Delta x_{t \tau}-i_{t \tau}-\tilde{m}\left(\pi_{t b}\right) f_{t \tau}, \tau>t \\
n_{(t+1) \tau} & =n_{t \tau}+\Delta n_{t \tau}, \tau>t \\
n_{t \tau} & =x_{t \tau}=0, \tau \leq t
\end{aligned}
$$

\section{B. Allowable Decisions and Feeder Constraints}

We are assuming that PEVs are not injecting power into the grid, but instead are modulating their consumption about an average rate in order to provide RS. Therefore, insurance that bids are realizable for each hour-ahead market period $t$ imposes two constraints on flexible and inflexible quantities. First, there must be sufficient feeder capacity (20). Note that this constraint couples all departure classes. Second, enough PEVs must be plugged-in (21), where $\bar{r}$ indicates the maximum charging rate of each PEV in $\mathrm{kW}$. Finally, (22) does not allow more energy to be charged than the current uncharged capacity.

$$
\begin{aligned}
\sum_{\tau}\left(i_{t \tau}+f_{t \tau}\right) & \leq C_{t} \\
i_{t \tau}+f_{t \tau} & \leq \bar{r} n_{t \tau}, \forall \tau \\
i_{t \tau}+f_{t \tau} & \leq x_{t \tau}, \forall \tau
\end{aligned}
$$

\section{Period Costs}

The period costs (23) are the sum of four terms. First, a marginal penalty for PEVs scheduled to depart that have not reached their desired SoC. Second, the expected cost of the inflexible energy bid. Third, the expected cost of the flexible energy bid conditioned on the bid being cleared as regulation service, market event FCRS. And fourth, the expected cost of the flexible bid conditioned on the bid being cleared as energy, market event FCE.

$$
\begin{aligned}
& E\left[g_{t}(\cdot)\right]=\phi x_{t(\tau=t)}+E\left[\tilde{\lambda}_{t}^{E}\right] \sum_{\tau} i_{t \tau}+ \\
& \quad \frac{1}{2} \operatorname{Pr}(F C R S) E\left[\tilde{\lambda}_{t}^{E}-\tilde{\lambda}_{t}^{R} \mid F C R S\right] \sum_{\tau} f_{t \tau}+ \\
& \quad \operatorname{Pr}(F C E) E\left[\tilde{\lambda}_{t}^{E} \mid F C E\right] \sum_{\tau} f_{t \tau}
\end{aligned}
$$

\section{Bellman Equation}

In order to formulate the Bellman Equation (27) concisely, we define vector notation in (24)-(26).

$$
\begin{aligned}
\tilde{\boldsymbol{\lambda}}_{t} & =\left[\tilde{\lambda}_{t}^{E}, \tilde{\lambda}_{t}^{R}\right] \\
\mathbf{x}_{t} & =\left[n_{t \tau}, x_{t \tau}\right] \\
\mathbf{u}_{t} & =\left[i_{t \tau}, f_{t \tau}, \pi_{t b}\right]
\end{aligned}
$$

The allowable control set, $U_{t}\left(\mathbf{x}_{t}\right)$, is defined by (20)-(22) as well as non-negativity constraints on the flexible and inflexible quantities and state variables. The boundary condition (28) is an end of cycle cost consisting of the sum of two terms. First, a marginal penalty cost $\phi$ assessed for any PEVs scheduled to depart at the end of the horizon (i.e., $\tau=24$ ) but not fully charged; and second, a marginal extension period cost $\mu_{\tau}$ per unit of uncharged energy of PEVs scheduled to depart outside the modeled cycle (i.e., $\tau>24$ ).

$$
J_{t}\left(\mathbf{x}_{t}\right)=\min _{\mathbf{u}_{t} \in U_{t}\left(\mathbf{x}_{t}\right)} E\left[g_{t}\left(\mathbf{x}_{t}, \mathbf{u}_{t}, \tilde{\boldsymbol{\lambda}}_{t}\right)+J_{t+1}\left(\mathbf{x}_{t+1}\right)\right]
$$

with boundary condition,

$$
J_{24}\left(\mathbf{x}_{24}\right)=\phi x_{24(\tau=24)}+\sum_{\tau>24} x_{24 \tau} \mu_{\tau} .
$$

It is important to note that the cost-to-go is a function of the state, emphasizing that the utility of PEV charging during period $t$ is a function of the expected future consumption trajectory as it will be determined by optimal future bidding.

\section{Solution Approach Adopted}

A full backward recursion solution of the proposed finite horizon stochastic dynamic program (SDP) is not tractable for any real system due to uncountable state and control spaces. Therefore, we approximate the SDP cost-to-go by a multistage stochastic programming (MSP) model. This modeling framework is scalable, in that it can handle the many departure classes that may occur at a particular feeder while limiting state space explosion.

\section{A. Multistage Stochastic Programming}

As shown in (17), uncertain market outcomes play a central role in the PEV energy dynamics, as the energy capacity state variables evolve according to market events associated with the flexible energy bid. Therefore, as in [20], we propose a forward-looking modeling framework that captures these uncertainties by approximating the SDP cost-to-go function using a MSP formulation [25].

The MSP model, depicted in Fig. 2, has an explicit lookahead which models future states using a scenario tree based on all possible market events or realizations of $\tilde{m}\left(\pi_{t b}\right)$. Each node has associated state and decision variables and the problem complexity grows exponentially in the number of time periods considered for the look-ahead. Therefore, we consider a limited look-ahead for a portion of the horizon (indicated by solid arrows) combined with a certainty equivalent for the remaining horizon (indicated by dashed arrows).

The SDP cost-to-go is approximated by minimizing the sum of the expected period costs at each node in the scenario tree, 


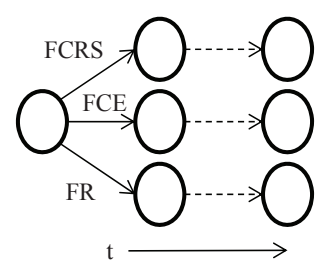

Fig. 2. Graphical depiction of forward looking MSP scenario tree.

weighted by the probability of visiting that node. The MSP solution indicates values for the decision variables at every node; however, as an optimal open loop feedback formulation, the only decisions implemented are those at the root node, or the first time period in the modeled horizon. The MSP is resolved in subsequent time periods.

The resulting MSP problem is non-linear and non-convex. To clarify, consider a component of the period costs (23),

$$
\operatorname{Pr}(F C E) E\left[\tilde{\lambda}_{t}^{E} \mid F C E\right] f_{t \tau} .
$$

Recall that the probability and conditional expected value of the flexible bid cleared as energy market event (FCE) are a function of the flexible bid price, $\pi_{t b}$, as we see in (10).

\section{B. Extension Period Cost Approximation}

The MSP hour-ahead formulation has equality constraints whose associated dual variables provide an estimate of the extension period costs, $\mu_{\tau}$. The constraints corresponding to the initial state of uncharged energy at the root node of the scenario tree, have dual variables that represent the marginal cost of an additional $\mathrm{kWh}$ of energy in departure class $\tau$ plugged-in at the beginning of the modeled cycle. Assuming that the modeled cycle is not drastically different from the subsequent daily cycle, these values provide a extension period cost approximation and motivate the Extension Period Estimation Algorithm presented in Fig. 3. Here we introduce the superscript $k$ as an iteration counter for the algorithm.

Step 0 initializes $k=1$ and sets the high initial estimate of

$$
\mu_{\tau}^{k}=\phi, \forall \tau>24 \text {. }
$$

Step 1 solves the MSP hour-ahead market problem using $\mu_{\tau}^{k}$ as the extension period costs. The new estimate of the extension period costs, $\mu_{\tau}^{k+1}$, is obtained using the optimal dual variables.

Step 2 checks the convergence of the estimation, and if

$$
\mu_{\tau}^{k+1}=\mu_{\tau}^{k}, \forall \tau>24,
$$

then the algorithm terminates and the solution is stored. Otherwise the iteration counter is incremented and the MSP hour-ahead market problem is resolved in Step 1.

In Theorem 1 we prove the algorithm converges given the reasonable assumption of finite clearing prices. Moreover, we show the convergence rate is a function of feeder congestion. The proof requires Claims 1-3.

Claim 1: The sequences $\left\{\mu_{\tau}^{k}\right\}$ are bounded below $\forall \tau>24$.

Proof: For iterations $k \geq 2, \mu_{\tau}^{k}$ is defined as the shadow price of the uncharged energy at the root node, scheduled to depart at $\tau-24$. This shadow price represents the cost of

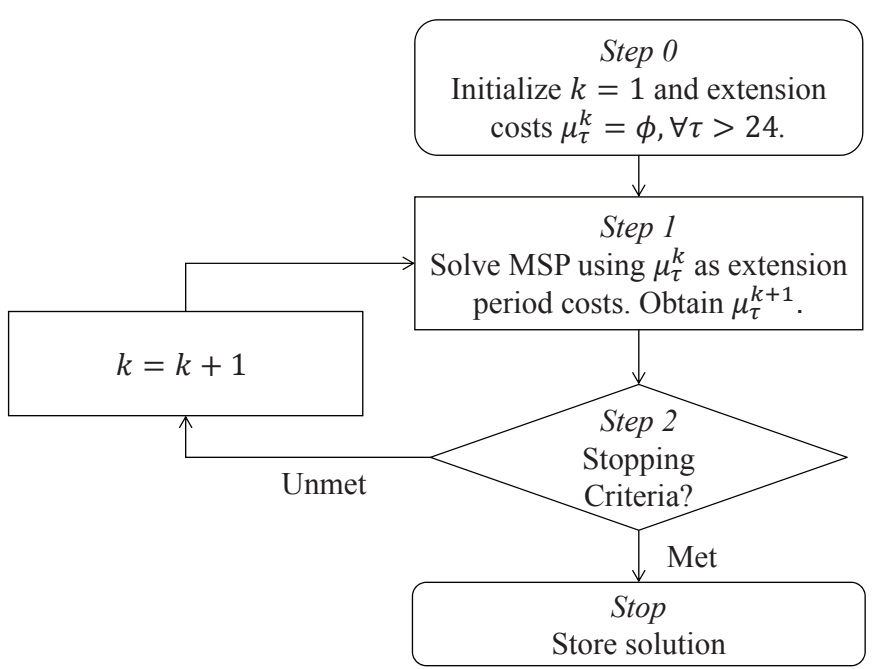

Fig. 3. Flow chart of the extension period estimation algorithm.

adding a marginal unit of demand with departure time $\tau-$ 24 at the beginning of the modeled cycle. PEVs are charged by providing RS or through energy consumption, or the LA is assessed a penalty, $\phi$, for not charging the PEV by the scheduled time of departure. Therefore, $\mu_{\tau}^{k}$ for $k \geq 2$ can never be less than

$$
\min \left\{\left\{E\left[\tilde{\lambda}_{t}^{E}-\tilde{\lambda}_{t}^{R}\right] \mid \forall t\right\} \cup\left\{E\left[\tilde{\lambda}_{t}^{E}\right] \mid \forall t\right\} \cup \phi\right\},
$$

which is finite due to our assumption of finite energy and RS clearing prices. Therefore, $\left\{\mu_{\tau}^{k}\right\}$ is bounded below as desired.

Claim 2: $\mu_{\tau}^{k+1} \leq \mu_{\tau}^{k} \Rightarrow x_{24 \tau}^{k+1} \geq x_{24 \tau}^{k}$, for all nodes s.t. $t=24, \forall \tau>24$.

Proof: Let $\mathbf{u}^{k *}$ be the optimal solution of the $k t h$ iteration. Since the feasible set does not change from iteration $k$ to $k+1$, the optimal solution $\mathbf{u}^{k *}$ is still feasible for the $(k+1) t h$ iteration. Therefore, by the optimality of $\mathbf{u}^{k *}$ and our given assumption that extension period costs are non-increasing we have the desired result. In other words, if the extension period costs decrease, then by the optimality of the previous iteration, only more uncharged energy will be left for the extension period in the next iteration.

Claim 3: The sequences $\left\{\mu_{\tau}^{k}\right\}$ are non-increasing $\forall \tau>24$.

Proof: We will use a proof by induction. Please recall that in Step 0 of the algorithm we have set

$$
\mu_{\tau}^{1}=\phi, \forall \tau>24 \text {. }
$$

If the energy and RS prices are such that charging a PEV would be more expensive than the penalty cost $\phi$, then the optimal decision will have the LA pay the penalty and not charge the PEVs prior to their departure time. Therefore, we get

$$
\mu_{\tau}^{2} \leq \phi=\mu_{\tau}^{1}, \forall \tau>24 .
$$

The next step in this inductive proof is to assume that,

$$
\mu_{\tau}^{k} \leq \mu_{\tau}^{k-1}, \forall \tau>24 .
$$

and show that this implies that

$$
\mu_{\tau}^{k+1} \leq \mu_{\tau}^{k}, \forall \tau>24 .
$$




\section{Given (29) we know that by Claim 2 \\ $\Rightarrow x_{24 \tau}^{k} \geq x_{24 \tau}^{k-1}$, for all nodes s.t. $t=24, \forall \tau>24$.}

Therefore, there are two possible outcomes to consider as we move from iteration $k-1$ to $k$.

- Case I: No change in the optimal charging decisions, meaning none of the energy has been moved to the extension periods. Formally, $x_{24 \tau}^{k}=$ $x_{24 \tau}^{k-1}$, for all nodes s.t. $t=24, \forall \tau>24$.

- Case II: Additional energy charging is left for the extension periods, or $x_{24 \tau}^{k}>x_{24 \tau}^{k-1}$ for some node and $\tau>24$.

If Case I occurs, then by the optimality of $\mathbf{u}^{(k-1) *}$ there will be no changes in the charging decisions for departure classes $\tau=1, \ldots, 24$ in $\mathbf{u}^{k *}$. And therefore,

$$
\mu_{\tau}^{k+1}=\mu_{\tau}^{k}, \forall \tau>24
$$

For Case II, the movement of some energy to the extension periods may alleviate feeder congestion. If no binding feeder constraint becomes unbinding, then by the optimality of $\mathbf{u}^{(k-1) *}$ there will be no changes in the charging decisions for departure classes $\tau=1, \ldots, 24$ in $\mathbf{u}^{k *}$. In addition, even if a binding distribution capacity constraint becomes unbinding, there may be no changes in the optimal charging decisions. In these instances

$$
\mu_{\tau}^{k+1}=\mu_{\tau}^{k}, \forall \tau>24
$$

However, if a binding feeder constraint becomes unbinding, then it may result in a change in the optimal charging decisions $\mathbf{u}^{(k-1) *}$ in iteration $k$. Let's assume this is the case for some $\tau^{\prime}$, such that $1 \leq \tau^{\prime} \leq 24$. Since $\mathbf{u}^{(k-1) *}$ remains feasible in iteration $k$, then by the optimality of $\mathbf{u}^{k *}$, this will only occur if the change reduces the expected cost for charging PEVs in departure class $\tau^{\prime}$. And therefore,

$$
\Rightarrow \mu_{\tau^{\prime}+24}^{k+1}<\mu_{\tau^{\prime}+24}^{k}
$$

This completes the proof by induction as we have considered Cases I-II.

Given the above claims, the convergence of the extension period cost estimation algorithm directly follows as shown in Theorem 1.

Theorem 1: $\left\{\mu_{\tau}^{k}\right\}$ are convergent sequences $\forall \tau>24$.

Proof: Given Claims 1 and 3, $\left\{\mu_{\tau}^{k}\right\}$ converge by the monotone convergence principle.

Corollary 1: The convergence rate of $\left\{\mu_{\tau}^{k}\right\}, \forall \tau>24$, is a function of the feeder congestion, $C_{t}$.

Proof: By the non-increasing property proved in Claim 3 , the algorithm converges in iteration $k$ unless

$$
\mu_{\tau}^{k+1}<\mu_{\tau}^{k}
$$

for some $\tau$. In the proof of Claim 3, we see that this only occurs if a feeder constraint was binding during iteration $k-1$. Therefore, if the feeder is particularly congested then the algorithm may take additional iterations to converge, establishing the dependence on $C_{t}$.

\section{Solving the Non-Linear MSP}

We employ iterative Gauss-Seidel inspired methods in order to solve the non-linear MSP [26]. Note that for fixed values of $\pi_{t b}$, the MSP reduces to a linear program (LP).

In Sections $\mathrm{V}$ and VI, we assume that the extension period estimation algorithm (Fig. 3) is employed in order to estimate the marginal costs of the subsequent daily cycle. We solve the non-linear MSP using an iterative technique where we fix bid prices, $\pi_{t b}$, and solve the resulting LP to obtain the optimal bid quantities, $i_{t \tau}, f_{t \tau}$ associated with the fixed bid prices. We then update bid prices and resolve for optimal quantities. Finally, we select the price-bid combination with the minimum expected cost-to-go.

In Section VII, we assume the LA has a good estimate of the extension period costs, and therefore, does not need to utilize the extension period cost algorithm. In this case, a faster gradient based approach may be employed limiting the number of LPs that must be solved.

Assume we fix the flexible bid prices to $\pi^{k}$, where $k$ is again the iteration counter, and solve the resulting LP to get bid quantities, $\mathbf{x}^{k}$ and expected cost $\mathcal{C}\left(\boldsymbol{\pi}^{k}\right)$. In the look-ahead portion of the MSP, the flexible bid prices do not impact the feasibility of the bid quantities, but only the LP cost vector, $\mathbf{c}^{k}$. Therefore, by perturbing the bid prices, updating the cost vector, $\mathbf{c}_{\text {per }}^{k}$, and evaluating at $\mathbf{x}^{k}$, we can quickly compute an estimate of the gradient as in (30).

$$
\nabla \mathcal{C}\left(\boldsymbol{\pi}^{k}\right)=\frac{\partial \mathcal{C}\left(\boldsymbol{\pi}^{k}\right)}{\partial \boldsymbol{\pi}^{k}}=\mathbf{c}_{p e r}^{\prime k} \mathbf{x}^{k}
$$

Note that the extension period costs must remain fixed for the gradient method to converge, as changing the extension period costs impacts the cost vector and invalidates the above estimate of the gradient.

Using this gradient estimate, we can employ a steepest descent gradient method [27] to update the bid prices as presented in (31),

$$
\boldsymbol{\pi}^{k+1}=\boldsymbol{\pi}^{k}-\alpha^{k} I \nabla \mathcal{C}\left(\boldsymbol{\pi}^{k}\right)
$$

where $I$ is the identity matrix and $\alpha^{k}$ is the stepsize. Selection of the stepsize to will be discussed in Section VII.

\section{CAse Study}

We modeled a 24 time period cycle with a second cycle extension period. The opportunity cost of uncharged batteries of PEVs departing during the second cycle is modeled as described above. The cycle begins at 12am on Day One, incorporates an estimated demand pattern, calculates charging decisions through 12am (Day Two), and estimates extension period costs for PEVs with departure times during the second cycle. The first three time periods comprise the look-ahead portion of the MSP horizon, while the remaining 19 time periods make up the certainty equivalent portion. We chose this look-ahead horizon so that explicit look-ahead extends to the first PEV departure class of 3 am (see Table I). A sensitivity analysis with respect to the length of the look-ahead horizon is presented in Section VI-A. The resulting MSP is an LP with approximately $65 \mathrm{k}$ state and decision variables and a 
similar number of constraints. The algorithm was implemented in Matlab, using the CPLEX 12.2 LP solver on a workstation with two $2.66 \mathrm{GHz}$ Intel Xeon processors and $24 \mathrm{~GB}$ of RAM.

Because it would be intractable to search over the entire range space of bid prices for each node in the scenario tree, we fix the price bids according to (32) for $t=2, \ldots, 24$. We then search for the optimal initial flexible price bid, $\pi_{1 b}$. The sub-optimality of this policy is discussed in Section VII.

$$
\pi_{t b}=E\left[\tilde{\lambda}_{t}^{E}\right]
$$

\section{A. Model Inputs}

We modeled a low voltage residential feeder servicing approximately fifty households. As in [20], we assumed that the feeder was rated for $10 \mathrm{~kW}$ per household $(500 \mathrm{~kW}$ total), and estimated the non-PEV load profile to determine available feeder capacity for PEV charging, $C_{t}$. Note that this is a simplification, as feeder capacity has a temporal component due to ambient air temperature variability and other considerations. In order to evaluate the impact of preexisting distribution network congestion, we modeled two distribution network scenarios corresponding to a nominal and high congestion scenarios as shown in Fig. 4.

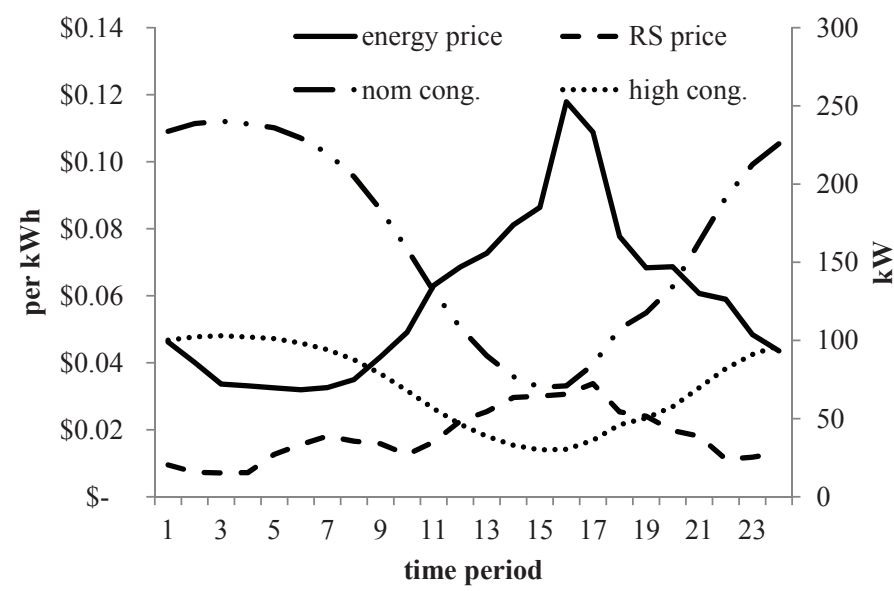

Fig. 4. Available feeder capacity and price means by time period.

Electricity market price probability distributions, available [28], were estimated using the maximum likelihood-ratio test applied to NYISO energy and RS prices from June to August 2011. Results for ERCOT and CAISO data are presented in [20]. Energy and RS price means by time period are presented in Fig. 4.

Similar to [20], we developed a simple PEV neighborhood demand pattern on which to test our market participation policy. This is intended as a proof of concept rather than a comprehensive analysis of all potential PEV demand patterns. As shown in the left column of Table I, we assumed that our neighborhood consisted of 100 PEVs, all of which are plugged-in at the beginning of the modeled cycle and scheduled to depart between $3 \mathrm{am}$ and $11 \mathrm{am}$. As shown on the right column of Table I, each of these PEVs is expected to return home in the afternoon or evening of Day One, with expected departure times in the morning of Day Two. We assume each of these PEVs has an empty battery when returning from a trip and assume that the owner requested $\mathrm{SoC}$ is $100 \%$. We evaluate the impacts of different battery capacities $(\mathrm{kWh})$ in Section VI-D. For the case study, we assumed a battery capacity of $6 \mathrm{kWh}$.

TABLE I

Estimated PEV DEMAND PATTERN

\begin{tabular}{ccc|ccc}
\hline \hline arr time & $\begin{array}{c}\text { dep time } \\
\text { day one }\end{array}$ & no & arr time & $\begin{array}{c}\text { dep time } \\
\text { day two }\end{array}$ & no \\
\hline before 12am & $3 \mathrm{am}$ & 2 & $2 \mathrm{pm}-3 \mathrm{pm}$ & $9 \mathrm{am}$ & 10 \\
before 12am & $4 \mathrm{am}$ & 3 & $2 \mathrm{pm}-3 \mathrm{pm}$ & $10 \mathrm{am}$ & 10 \\
before 12am & $5 \mathrm{am}$ & 10 & $2 \mathrm{pm}-3 \mathrm{pm}$ & $11 \mathrm{am}$ & 5 \\
before 12am & $6 \mathrm{am}$ & 10 & $3 \mathrm{pm}-4 \mathrm{pm}$ & $6 \mathrm{am}$ & 25 \\
before 12am & $7 \mathrm{am}$ & 25 & $4 \mathrm{pm}-5 \mathrm{pm}$ & $6 \mathrm{am}$ & 20 \\
before 12am & $8 \mathrm{am}$ & 25 & $5 \mathrm{pm}-6 \mathrm{pm}$ & $7 \mathrm{am}$ & 10 \\
before 12am & $9 \mathrm{am}$ & 10 & $6 \mathrm{pm}-7 \mathrm{pm}$ & $7 \mathrm{am}$ & 10 \\
before 12am & $10 \mathrm{am}$ & 10 & $7 \mathrm{pm}-8 \mathrm{pm}$ & $8 \mathrm{am}$ & 10 \\
before 12am & $11 \mathrm{am}$ & 5 & & & \\
\hline \hline
\end{tabular}

As in [20], uncharged capacity at the requested time of departure was penalized at $\$ 0.75$ per $\mathrm{kWh}, \phi$, which assumes $\$ 3.75$ per gallon of gasoline, 25 miles per gallon, and 5 miles per $\mathrm{kWh}$. In addition, each PEV was assumed to have a maximum charging rate, $\bar{r}$, of $4 \mathrm{~kW}$. Sensitivity analysis with regards to these parameters are presented in Sections VI-B and VI-C, respectively.

\section{B. Case Study Results}

We compare the results for three possible PEV charging policies. First, we evaluate the policy proposed in this paper, namely market participation. Second, we consider selfscheduling, where a LA only submits inflexible energy bids $l_{t b}$. Note this is equivalent to the LA charging batteries based on a forecast of electricity prices. For both market participation and self-scheduling we assume that the LA observes the feeder capacity constraint (20). Finally, the cost of opportunity charging, namely the cost of charging immediately upon plugging-in until the desired state-of-charge is reached regardless of available feeder capacity, is calculated using time dependent electricity costs. Column 1 of Table II presents the benchmark case of opportunity charging. Columns 2-3 show the nominal congestion scenario and columns 4-5 the high congestion scenario for the self-scheduling and market participation policies. The expected flexible bids row indicates the expected percentage of all market bids which are flexible, while the expected $R S$ energy row indicates the expected percentage of energy consumed while providing RS.

The self-scheduling and market participation policies result in significant cost savings. By allowing flexible bids, the cost savings increased by approximately $15 \%$ in the nominal congestion scenario and $5 \%$ in the high feeder congestion scenario. For the market participation policy, the increased feeder congestion decreases the percentage of expected flexible bids by more than $10 \%$ and the expected energy from RS provision by $20 \%$. 
TABLE II

CASE Study Results Summary

\begin{tabular}{|c|c|c|c|c|c|}
\hline \multirow{3}{*}{ metric } & \multirow{3}{*}{$\begin{array}{c}\text { opportunity } \\
\text { charging }\end{array}$} & \multicolumn{2}{|c|}{ nominal congestion } & \multicolumn{2}{|c|}{ high congestion } \\
\hline & & self- & market & self- & market \\
\hline & & scheduling & participation & scheduling & participation \\
\hline expected total cost $(\$)$ & 71.57 & 39.00 & 28.19 & 44.05 & 40.10 \\
\hline expected savings $(\%)$ & $\mathrm{n} / \mathrm{a}$ & 45.50 & 60.62 & 38.46 & 43.98 \\
\hline scenario $\min / \max$ expected total cost & $\mathrm{n} / \mathrm{a}$ & $\mathrm{n} / \mathrm{a}$ & $21.89 / 29.22$ & $\mathrm{n} / \mathrm{a}$ & $37.25 / 41.67$ \\
\hline expected incremental cost ( $\$$ per $\mathrm{kWh})$ & 0.060 & 0.032 & 0.023 & 0.037 & 0.033 \\
\hline expected flexible bids $(\%)$ & 0.00 & $\mathrm{n} / \mathrm{a}$ & 92.21 & $\mathrm{n} / \mathrm{a}$ & 79.19 \\
\hline expected RS energy (\%) & 0.00 & $\mathrm{n} / \mathrm{a}$ & 55.88 & $\mathrm{n} / \mathrm{a}$ & 36.47 \\
\hline extension period estimation algorithm iterations & $\mathrm{n} / \mathrm{a}$ & 2 & 3 & 2 & 6 \\
\hline computation time $(\mathrm{s})$ & $\mathrm{n} / \mathrm{a}$ & 0.19 & 0.94 & 0.22 & 1.903 \\
\hline
\end{tabular}

The extension period approximation algorithm took three additional iterations to converge due the increased feeder congestion, validating Corollary 1. The impacts of extension period costs are discussed in the individual sensitivity analyses and in particular in Fig. 9.

Fig. 5 shows the expected cost-to-go as a function of the period 1 flexible price bid for the nominal and high congestion scenarios. The function has an optimum at an initial bid price of $\$ 0.03$ for the nominal congestion scenario, which is below the expected energy price of $\$ 0.05$ for $12 \mathrm{am}-1 \mathrm{am}$. By selecting a bid price below the expected value, the LA is taking a risk that it may not receive the energy, but if it does, it will incur a lower cost. At an initial flexible bid price of $\$ 0.03$, there is a 0.20 probability of the flexible bid cleared as RS market event, and only a 0.04 probability of the flexible bid cleared as energy market event; however, the expected costs are $\$ 0.0167$ and $\$ 0.0003$, respectively. In the high congestion scenario, the optimal initial flexible bid price is $\$ 0.06$, which is greater than the expected energy price for $12 \mathrm{am}-1 \mathrm{am}$. By increasing the initial bid price in the high congestion scenario, the LA is placing a premium on feeder capacity, as the probability it receives the energy increases but the expected costs do as well. At a bid price of $\$ 0.06$, the before mentioned probabilities increase to 0.40 and 0.48 , respectively; however, the expected costs increase to $\$ 0.0408$ and $\$ 0.0323$, respectively.

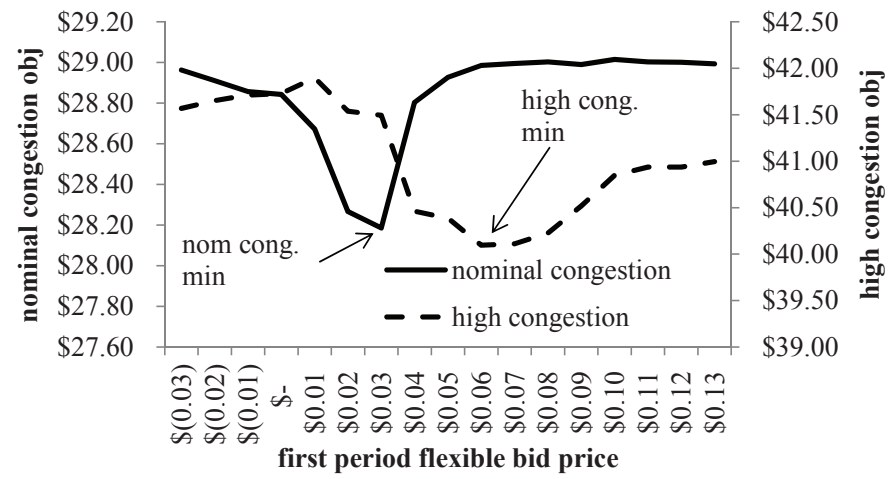

Fig. 5. Expected cost-to-go as a function of first period flexible price bid.

As presented in [20], the market participation policy presents the best option for valley filling, a fact corroborated by Fig. 6. Given the assumed demand pattern, opportunity charging increases and extends peak power consumption by several hours, and the feeder capacity constraint is violated. As suggested in [20], it may be possible for the LA to negotiate discounted distribution network charges for limiting feeder wear and tear by observing a capacity constraint in the market participation and self-scheduling policies. Self-scheduling will shift the charging to times of low feeder congestion; however, charging will gravitate to a few hours resulting in a second peak in the load profile, sometimes referred to as a rebound peak. A similar result is reported in [18], [20]. For the market participation policy, due to the uncertainty in the market events with respect to the flexible bids, and the fact PEVs must charge below their maximal consumption rate in order to provide RS, there is an incentive to start charging earlier and at a lower rate.

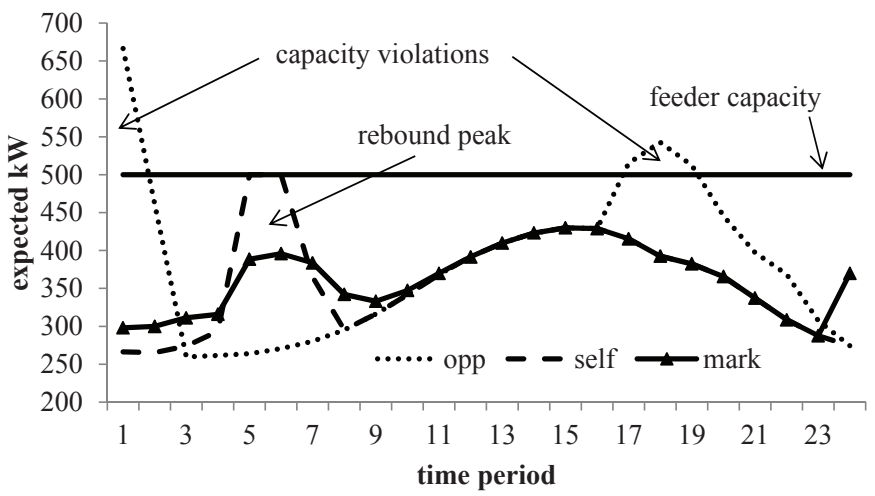

Fig. 6. Expected load profile by PEV charging policy.

Fig. 7 shows the expected aggregate uncharged energy and flexible and inflexible bids for the market participation policy under nominal feeder congestion. In the morning, the LA enters inflexible bids to ensure all PEVs have reached their desired SoC by their scheduled departure time. In the afternoon, only flexible bids are entered in order to avoid extension period costs.

\section{SEnsitivity AnAlysis}

In this section, we examine the sensitivity of our case study results to specific model parameters including the length of the look-ahead horizon, maximum PEV charging rate, penalty cost, and PEV battery capacity. 


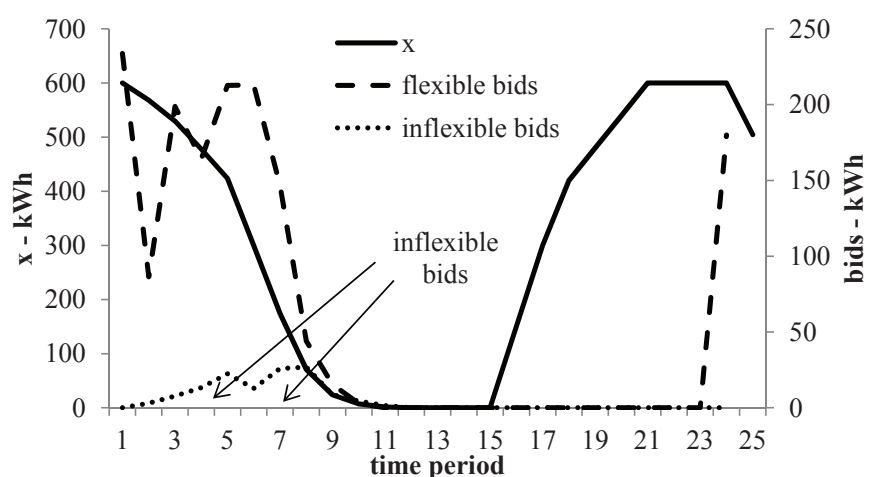

Fig. 7. Market participation expected quantity bids and uncharged energy.

\section{A. Look-Ahead Sensitivity}

In the case study presented in Section V-B, we included three periods in the look-ahead horizon, and 19 periods in the certainty equivalent. A full look-ahead formulation ensures all PEVs are charged prior to their scheduled departure time, for all possible market outcomes, when this is physically feasible and economically desirable. In a certainty equivalent formulation, market outcomes with positive probability may result in uncharged energy at the time of PEV departure and associated penalty costs.

We examined explicit look-ahead horizons of length one through four to determine the impacts on the initial bidding policy. Interestingly, results showed that the initial bid price of $\$ 0.03$ was consistent for all length of look-ahead horizons. In addition, aggregate initial flexible and inflexible bid quantities, constrained by the available feeder capacity, were consistent across all look-ahead horizons. However, the allocation of the initial flexible bid quantity across departure classes varied. In general, shorter look-ahead horizons resulted in more aggressive bidding strategies, where energy is not allocated exclusively to the nearest departure classes. Longer look-ahead horizons are better able to account for low probability, high cost scenarios where consecutive flexible bid rejected market events occur resulting in PEV departures prior to reaching their desired SoC. Computation time increases exponentially according to the length of the look-ahead horizon; although, since the resulting model is an LP, the computation time is still reasonable, where a look-ahead horizon of four can be solved in approximately eight seconds.

\section{B. Penalty Cost Sensitivity}

The penalty cost, $\phi$, of PEVs that do not reach their desired SoC by the time of scheduled departure should depend on the characteristics of the PEV as well as user preference. For example, uncharged batteries at the time of departure for an all-electric vehicle should be costlier than uncharged energy for a hybrid electric vehicle. In addition, an environmentally conscious driver may place a high premium on using electricity in preference to gasoline. The purpose of the penalty cost is to ensure that PEVs reach their desired SoC by their scheduled departure time, given that it is physically feasible and economically desirable. Higher penalty costs result in more risk averse bidding, either through more inflexible bids, see Fig. 8, or higher initial flexible bid prices; however, we found the optimal flexible and inflexible bids converged for relatively low values of $\phi$.

The optimal initial flexible bid price was $-\$ 0.03$ for $\phi=$ $\$ 0.00, \$ 0.02$ for $\phi=\$ 0.01$, and $\$ 0.03$ for $\phi \geq \$ 0.02$. The initial optimal flexible and inflexible bid quantities converge for all values of $\phi \geq \$ 0.06$. As shown in Fig. 8, due to the relative cheap costs of electricity, low values of $\phi$ resulted in identical expected bidding policies as those for $\phi=\$ 0.75$.

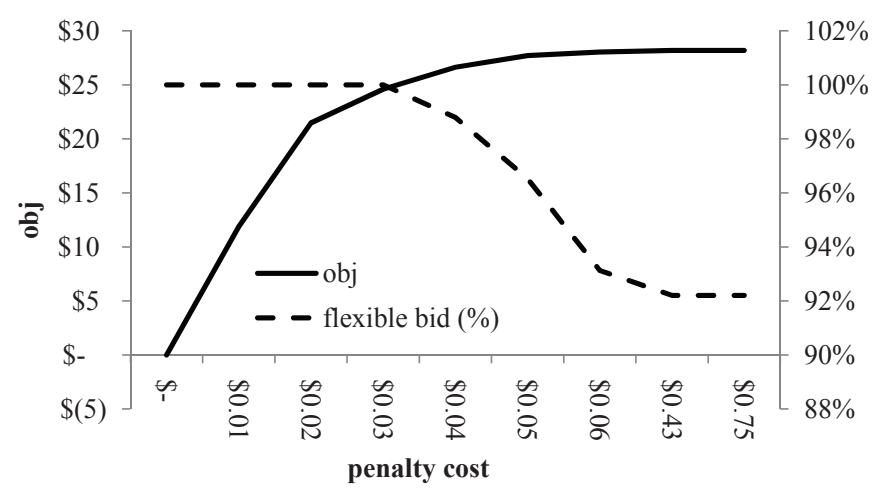

Fig. 8. Expected cost and initial bid price as a function of penalty cost.

\section{Maximum Charge Rate Sensitivity}

Case study results, presented in Section V-B, show that the maximum charging rate of each $\mathrm{PEV}, \bar{r}$, is binding for several departure classes and time periods. Therefore, we performed a sensitivity analysis on the maximum charge rate in order to determine its impact on the PEV charging costs and expected optimal bids. As shown in Table III the expected charging cost decreases as $\bar{r}$ increases, until $\bar{r}=8$, where the vehicle charging rates are no longer binding in any scenarios. Cost savings are due to increased expected energy consumed through RS provision, which increases from $47 \%$ to $58 \%$.

TABLE III

Maximum PEV Charge Rate Sensitivity Results

\begin{tabular}{ccc|ccc}
\hline \hline $\mathrm{r}$ & obj & \%RS & $\mathrm{r}$ & obj & \%RS \\
\hline 2 & 32.89 & 47.28 & 6 & 27.71 & 57.93 \\
4 & 28.19 & 55.88 & 8 & 27.61 & 57.93 \\
\hline \hline
\end{tabular}

The maximum PEV charging rate impacts the extension period costs; and therefore, the LA charging decisions between the afternoon arrivals and the end of the first cycle (or 12am Day Two). Fig. 9, shows that when $\bar{r}=2$, the PEV maximum charging rate constrains the expected flexible bid quantities from 12am-9am. As a result, the extension period costs are higher for $\bar{r}=2$ than $\bar{r}=8$. Therefore, the LA chooses to place flexible bids in the evening of Day One in order to avoid high extension period costs.

\section{Battery Capacity Sensitivity}

Sensitivity analysis results with respect to PEV battery energy capacity $(\mathrm{kWh})$ are presented in Table IV. Increasing the battery capacity from $6 \mathrm{kWh}$ used in the case study to 16 


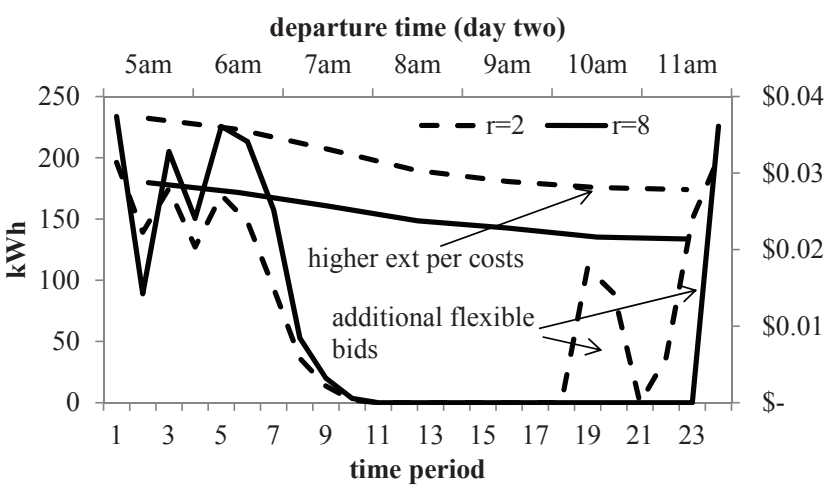

Fig. 9. Expected RS bids and extension period costs by max charge rate.

$\mathrm{kWh}$, which is the capacity of a Chevy Volt [29], increased the expected cost more than $300 \%$. Recall that flexible bids may be rejected or cleared as RS as a result of the market clearing. In these cases, the LA receives less energy than the maximum indicated by the allowable control set. With high battery capacities, energy is at a premium, so it is optimal for the LA to submit more inflexible bids at a higher expected cost, rather than risk consuming less energy and incurring a penalty cost. Therefore, the percentage of inflexible bids increases from $8 \%$ to $33 \%$. In addition, the optimal initial bid price increased from $\$ 0.03$ in the case study to $\$ 0.04$ with a $8 \mathrm{kWh}$ battery capacity, $\$ 0.05$ with a 10 or $12 \mathrm{kWh}$ battery capacity, and $\$ 0.06$ with a 14 or $16 \mathrm{kWh}$ battery capacity. This is consistent with the risk averse bidding observed in the penalty cost sensitivity analysis.

TABLE IV

BATTERY CAPACITY SENSITIVITY RESULTS

\begin{tabular}{ccc|ccc}
\hline \hline $\begin{array}{c}\text { bat } \\
\text { cap }\end{array}$ & obj & $\begin{array}{c}\text { inflexible } \\
\text { bids (\%) }\end{array}$ & $\begin{array}{c}\text { bat } \\
\text { cap }\end{array}$ & obj & $\begin{array}{c}\text { inflexible } \\
\text { bids (\%) }\end{array}$ \\
\hline 6 & 28.19 & 7.79 & 12 & 74.53 & 18.06 \\
8 & 41.89 & 9.95 & 14 & 97.95 & 25.13 \\
10 & 57.73 & 12.23 & 16 & 123.49 & 32.50 \\
\hline \hline
\end{tabular}

The expected flexible and inflexible bid quantities as well as the aggregate uncharged energy capacity for the case with battery capacities of $16 \mathrm{kWh}$ are shown in Fig. 10. As mentioned above, in order to avoid high penalty costs a significant amount of inflexible bids are submitted in the morning hours, while in the afternoon additional flexible bids occur relative to Fig. 7 results to avoid high extension period costs.

\section{GRadient Method Results}

In this section, we employ the gradient method presented in (31) in order to evaluate the sub-optimality of the bidding policy (32) used in Sections V and VI. Therefore, we fix the extension period cost estimates. In order to obtain a good estimate, initial bid prices are set to the energy price means, we solve the resulting LP, and employ the extension period estimation algorithm to obtain good extension period costs estimates, which we then fix during the gradient method application. The stepsize is selected during each iteration so

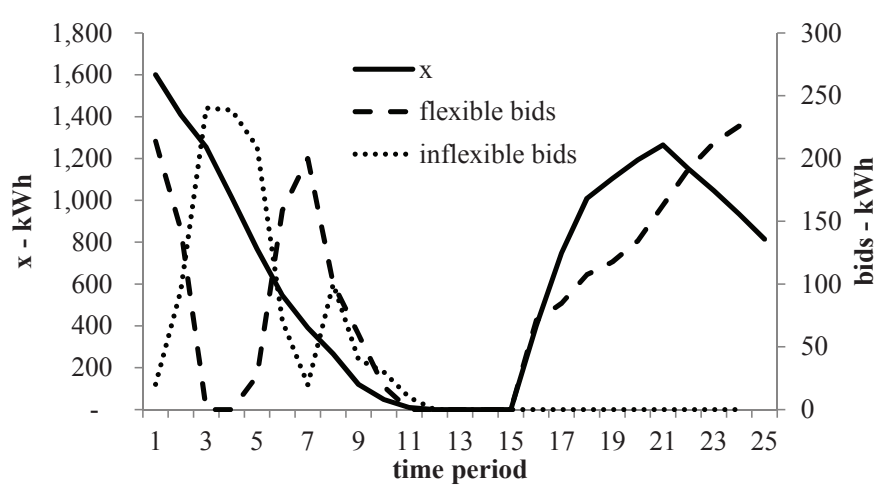

Fig. 10. Flexible policy expected bids and uncharged energy by time period for battery capacity of $16 \mathrm{kWh}$.

that the maximum change in the flexible bid price is $\$ 0.01$, and the stepsize is reduced if an iteration yields an increase in expected cost [27].

Table $\mathrm{V}$ presents the results of the gradient method. The first column indicates the number of time periods for which the flexible bid prices were optimized. Due to the fact that price bid decision variable exist for each node of the scenario tree (Fig. 2), the number of bid prices optimized grows exponentially in the number of time periods considered, as shown in the second column. The expected cost savings of optimally determining the bid prices decreases as the optimization extends to future time periods. Optimizing the first time period bid prices results in an expected cost savings of $1.24 \%$. Extending this to the second time period saves an expected $0.97 \%$, and to the third time period only $0.29 \%$.

TABLE V

GRADIENT METHOD RESULTS

\begin{tabular}{ccccc}
\hline \hline$t$ & \# prices & iterations & obj & change (\%) \\
\hline 0 & 0 & n/a & 28.8026 & n/a \\
1 & 1 & 2 & 28.4442 & 1.24 \\
2 & 4 & 5 & 28.1661 & 2.21 \\
3 & 13 & 32 & 28.0832 & 2.50 \\
\hline \hline
\end{tabular}

It is important to note that although the expected cost changes, the initial price and quantity bids do not change as we optimize the bid prices for future time periods. Therefore, in our optimal open loop feedback formulation, the implemented policy appears to be unaffected by the bidding policy in (32). However, this result is specific to the case study presented in this paper for three reasons. First, we used discrete probability distributions [28]; second, we chose a reasonable price bid policy in (32); and third, the inputs used in the case study result in the first period being non-marginal.

Assume that rather than the bidding policy described in (32), the price bids for $t \geq 2$ are instead set equal to the high price of $\$ 0.5$ per $\mathrm{kWh}$. Given this price bidding policy, the flexible bids are equivalent to fixed bids, the FCE market event occurs almost certainly, and no additional information derived with regards to the expected clearing prices conditional on the FCE market event. Using this sub-optimal bidding strategy for $t \geq 2$, the optimal first period bid price increases to $\$ 0.05$, as 
compared to $\$ 0.03$ for the near-optimal first period price bid presented in Section V. This premium results from LA desiring a high probability of the FCE or FCRS market events, and the associated lower conditional expected cost, in the one period it has the ability to do so.

Therefore, if the LA has a reasonable estimate of the extension period costs, or has few PHEVs leaving outside the modeled horizon, then using the gradient method to determine optimal price bids is preferable to approximating them. In fact, as we have done here, a reasonable extension period cost estimate can be computed using the extension period cost estimation algorithm and then fixed for the gradient method computations.

By examining optimal bid prices, we can gain intuition for general rules on the optimal flexible price bids. For example, if previous time periods realized market events have been flexible bid cleared as energy (FCE) or flexible bid cleared as RS (FCRS), the price bids can be more aggressive, i.e., lower. In particular, if consecutive FCE market events occur, then the optimal bid prices are much lower than the expected energy price, as the LA has already received its maximum quantity in the previous time periods. However, if the realized market events have been flexible bid rejected (FR), than the optimal bid price is higher in order to increase the probability of being scheduled in the current time period. Finally, it appears that price bids should be set below the expected energy price, given the nominal congestion scenario evaluated in this section.

\section{CONCLUSION AND FUtURE WORK}

We developed a market participation policy for a LA managing the charging of PEVs connecting at the same distribution network feeder. Our case study indicates that the market participation policy has the potential to substantially reduce daily electricity costs for PEVs as compared to opportunity charging. In addition, we found that by acting to account for the uncertainty of future power market clearing prices, rather than acting certainty equivalent point estimate may result in additional cost reductions. Finally, our market participation policy provides ISOs the opportunity to clear PEV demand in a manner that most benefits renewable generation, as flexible bid price-quantity pairs can be considered simultaneously for all reserve and load following functions, rather than being targeted for a specific type of reserves.

Future research will build on our hour-ahead market framework to examine a systematic coupling between existing wholesale and developing dynamic retail markets and the potential impacts of allowing load-side complex-bids (e.g., bids that include inter-temporal constraints) in day-ahead markets [30]. In addition, since we use a MSP to approximate the SDP cost-to-go, future work will evaluate the sub-optimality of the policies derived from the MSP formulation. In preliminary work, we evaluated a single PEV with an uncharged energy of $6 \mathrm{kWh}$ at $12 \mathrm{am}$ and scheduled to depart at $5 \mathrm{am}$. The MSP result suggests a conservative policy of an initial flexible bid of $4 \mathrm{kWh}$ at a price of $\$ 0.03$ for $12 \mathrm{am}-1 \mathrm{am}$. The SDP indicates that a more aggressive policy is optimal, were the LA does not bid until 2am-3am; however, the bid during that time period is the same as that derived from the MSP. In addition, future work will analyze the robustness of optimal bids to unexpected changes in the PEV demand pattern, and relax the energy neutrality assumption on the RS signal. Finally, as we mentioned in [20], we hope to show that this methodology can be applied to other types of demand such as space conditioning systems and data centers.

\section{REFERENCES}

[1] "National action plan on demand response," FERC, Tech. Rep. Docket No. AD09-10, Jun. 2010.

[2] H. Khurana, M. Hadley, N. Lu, and D. Frincke, "Smart-grid security issues," Security Privacy, IEEE, vol. 8, no. 1, pp. 81-85, jan.-feb. 2010.

[3] C.-L. Su and D. Kirschen, "Quantifying the effect of demand response on electricity markets," IEEE Trans. Power Syst., vol. 24, no. 3, pp. 1199-1207, Aug. 2009.

[4] R. Walawalkar, S. Fernands, N. Thakur, and K. R. Chevva, "Evolution and current status of demand response in electricity markets: Insights from PJM and NYISO," Energy, vol. 35, pp. 1553-1560, Apr. 2010.

[5] J. A. P. Lopes, F. J. Soares, and P. M. R. Almeida, "Integration of electric vehicles in the electric power system," Proc. IEEE, vol. 99, no. 1, pp. 168-182, Jan. 2011

[6] W. Kempton and J. Tomic, "Vehicle-to-grid power implementation: From stabilizing the grid to supporting large-scale renewable energy," J. Power Sources, vol. 144, pp. 268-279, Jun. 2005.

[7] M. C. Caramanis and J. M. Foster, "Coupling of day-ahead and real-time power markets for energy and reserves incorporating local distribution network costs and congestion," in Proc. 48th Allterton Conf. Comm., Cont., Comput., Sep. 2010, pp. 42-49.

[8] J. C. Smith, M. R. Milligan, E. A. DeMeo, and B. Parsons, "Utility wind integration and operating impact state of the art," IEEE Trans. Power Syst., vol. 22, no. 3, pp. 900-908, Aug. 2007.

[9] Y. Makarov, C. Loutan, J. Ma, and P. de Mello, "Operational impacts of wind generation on California power systems," IEEE Trans. Power Syst., vol. 24, no. 2, pp. 1039-1050, May 2009.

[10] L. Xie, P. M. S. Carvalho, L. A. F. M. Ferreira, J. Liu, B. H. Krogh, N. Popli, and M. D. Ilić, "Wind integration in power systems: Operational challenges and possible solutions," Proc. IEEE, vol. 99, no. 1, pp. 214-232, Jan. 2011.

[11] P. Denholm and W. Short, "An evaluation of utility system impacts and benefits of optimally dispatched plug-in hybrid electric vehicles," NREL, Tech. Rep. NREL/TP-620-40293, Oct. 2006.

[12] A. Y. Saber and G. K. Venayagamoorthy, "Efficient utilization of renewable energy sources by gridable vehicles in cyber-physical energy systems," IEEE Syst. J., vol. 4, no. 3, pp. 285-293, Sep. 2010.

[13] S. B. Peterson, J. F. Whitacre, and J. Apt, "Net air emissions from electric vehicles: The effect of carbon price and charging strategies," Environ. Sci. Tech., vol. 45, no. 5, pp. 1792-1797, Feb. 2011.

[14] S. Rahman and G. Shrestha, "An investigation into the impact of electric vehicle load on the electric utility distribution system," IEEE Trans. Power Delivery, vol. 8, no. 2, pp. 591-597, Apr. 1993.

[15] F. Li, N. P. Padhy, J. Wang, and B. Kuri, "Cost-benefit reflective distribution charging methodology," IEEE Trans. Power Syst., vol. 23, no. 1, pp. 58-64, Feb. 2008.

[16] A. D. Dominguez-Garcia and C. N. Hadjicostis, "Coordination and control of distributed energy resources for provision of ancillary services," in Proc. 1st IEEE Conf. Smart Grid Comm., Oct. 2010, pp. 537-542.

[17] K. Clement-Nyns, E. Haesen, and J. Driesen, "The impact of charging plug-in hybrid electric vehicles on a residential distribution grid," IEEE Trans. Power Syst., vol. 25, no. 1, pp. 371-380, Feb. 2010.

[18] Z. Ma, D. Callaway, and I. Hiskens, "Decentralized charging control for large populations of plug-in electric vehicles," in Proc. 49th IEEE Conf. Dec. Control, Dec. 2010, pp. 206-212.

[19] N. Rotering and M. Ilic, "Optimal charge control of plug-in hybrid electric vehicles in deregulated electricity markets," IEEE Trans. Power Syst., vol. 26, no. 3, pp. 1021-1029, Aug. 2011.

[20] J. M. Foster and M. C. Caramanis, "Energy reserves and clearing in stochastic power markets: The case of plug-in hybrid electric vehicle battery charging," in Proc. 49th IEEE Conf. Dec. Control, Dec. 2010, pp. 1037-1044.

[21] S. Han, S. Han, and K. Sezaki, "Development of an optimal vehicleto-grid aggregator for frequency regulation," IEEE Trans. Smart Grid, vol. 1, no. 1, pp. 65-72, Jun. 2010. 
[22] E. Sortomme and M. A. El-Sharkawi, "Optimal charging strategies for unidirectional vehicle-to-grid," IEEE Trans. Smart Grid, vol. 2, no. 1, pp. 131-138, Mar. 2011.

[23] "Ancillary services manual," NYISO, Tech. Rep., May 2011. [Online]. Available: http://www.nyiso.com/

[24] "Energy and ancillary services market operations," PJM, Tech. Rep., Oct. 2011. [Online]. Available: http://pjm.com/

[25] A. Ruszcynski and A. Shapiro, Stochastic Programming Handbooks in Operations Research and Management Science. Elsevier, 2003, vol. 10.

[26] B. Wollenberg and A. Wood, Power Generation, Operation and Control, 2nd ed. New York, NY: John Wiley, 1996.

[27] D. P. Bertsekas, Nonlinear Programming, 2nd ed. Belmont, MA: Athena Scientific, 1999.

[28] J. M. Foster. (2011, Nov.) Supporting information. [Online]. Available: http://people.bu.edu/jfoster2/research.html

[29] GM. (2011, Nov.) Chevy volt specifications. [Online]. Available: http://gm-volt.com/full-specifications/

[30] M. C. Caramanis and J. M. Foster, "Uniform and complex bids for demand response and wind generation scheduling in multi-period linked transmission and distribution markets," in Proc. 50th IEEE Conf. Dec. Control, Dec. 2011.

Justin M. Foster (S'09) received a B.A. in mathematics from Bowdoin College, Brunswick, Maine, in 2003, and a M.S. and Ph.D. in Systems Engineering at Boston University, Boston, Massachusetts, in 2011 and 2012, respectively. He is an EPA Science to Achieve Results (STAR) Fellow in Environmental Behavior and Decision Making and a Switzer Fellow. His research interests include tractable transmission topology control, sustainable power systems, electricity market design, and demand-side decision support.

Michael C. Caramanis (M'79) received his M.S. in Ch.E. from Stanford University and M.S. and Ph.D. in Engineering from Harvard University. He is a Boston University Professor of Mechanical and Systems Engineering, teaches in the areas of stochastic control, supply chains, and power markets, and conducts research in sustainable advanced building design and operation, control of demand and distributed resources in the presence of intermittent renewable generation, and the extension of power markets to include distribution costs and congestion with load participating on a par basis to generation. 